\title{
USO DE FATOR VII RECOMBINANTE ATIVADO PARA TRATAMENTO E PROFILAXIA DE GRANDES SANGRAMENTOS
}

\author{
Use of recombinant activated factor VII for treatment and prophylaxis of major \\ bleeding
}

\author{
Flávio Augusto Henriques VINCE, Maria José Nascimento BRANDÃO
}

ABCDDV/665

Vince FAH, Brandão MJN. Uso de fator VII recombinante ativado para tratamento e profilaxia de grandes sangramentos. ABCD Arq Bras Cir Dig 2009;22(3):171-8

RESUMO - Introdução - O fator VII recombinante ativado (rFVIIa) é uma proteína produzida por engenharia genética, cuja estrutura é muito semelhante à do fator VII intrínseco ativado (FVII). Sua ação se baseia no conhecimento do mecanismo de coagulação in vivo agindo na ativação direta do fator X resultando em formação independente de trombina no local da lesão e contribuindo dessa forma para formação de coágulo estável de fibrina sem a ação dos fatores VIII e IX. Métodos - Foi realizada ampla revisão da literatura com o objetivo de determinar os achados recentes relacionados ao uso de fator VII recombinante ativado em pacientes com sangramento severo. Resultados - Constatou-se que o uso de rFVIIa foi iniciado na década de 80 para profilaxia e tratamento de sangramento em pacientes com antecedente de hemofilia A ou B com inibidores de fator VIII e IX, deficiência de fator VII e trombastenia de Glanzmann refratários à reposição plaquetária. Em 1999 seu uso foi ampliado para outras situações clínicas e dessa forma, começaram a ser publicados diversos trabalhos mostrando a eficácia do rFVIIa como agente pró-hemostático em paciente com outras coagulopatias ou pacientes previamente hígidos com história de sangramento agudo de grande monta. Trauma é a principal causa de mortalidade no mundo sendo sangramento incontrolado o principal desafio no atendimento a estes pacientes. É comum a associação de trauma com coagulopatia, necessitando em algumas situações de terapia específica para o tratamento da mesma. Neste momento a terapia adjuvante com rFVIIa deve ser considerada. Outras causas comuns de sangramento são as operações cardíacas e ginecológicas/ obstétricas e doenças envolvendo o fígado. A coagulopatia nesses casos ocorre por deficiência dos fatores dependentes de vitamina K, sendo o FVII o com menor meia-vida. Conclusão - O uso de rFVIIa tem sido sugerido como opção terapêutica promissora para esses pacientes. Dessa forma, o recente aumento do uso de rFVIIa em situações ainda não aprovadas levou ao crescente questionamento sobre eficácia e segurança desta específica medicação em tais situações.

DESCRITORES - Fator VII. Hemorragia.

\section{INTRODUÇÃO}

O fator racombinante VII ativado (rFVIIa) é a forma recombinante do fator VII ativado produzida a partir da clonagem do gene humano do fator VII (FVII) transfectado para rins de fetos de hamsters; o DNA recombinante é proteoliticamente convertido em sua forma ativa, cuja estrutura é muito semelhante à do FVII ativado intrínseco, e tem meia-vida de duas horas ${ }^{19}$. Sua ação se baseia no conhecimento do mecanismo de coagulação in vivo, no qual o FVII intrínseco liga-se ao fator endotelial (FT), exposto no subendotélio após a injuria vascular, sendo ativado resultando na ativação de fator IX (FIXa) e na formação de pequena quantidade de trombina (conversão de protrombina em trombina) que irá ativar fator $\mathrm{V}(\mathrm{FVa})$ e VIII (FVIIIa), assim como plaquetas acumuladas na superfície da lesão levando à ativação do fator $\mathrm{X}(\mathrm{FX})^{1,10}$. O FX ativado (FXa) na superfície das plaquetas, interage com o FVa, cálcio e fosfolipídeos, levando à formação de

Trabalho realizado no Departamento de Anestesiologia da Faculdade de Ciências Médicas da Universidade Estadual de Campinas - UNICAMP, Campinas, SP, Brasil

Correspondência: Maria José Nascimento Brandão, e-mail: mjnbceu@fcm.unicamp.br grande quantidade de trombina (burst de trombina), o que resulta na conversão de fibrinogênio em fibrina à formação de coágulo de fibrina estável e à formação de inibidores de fibrinólise ativados por fibrina (TAFI ${ }^{1,10}$. Além de converter fibrinogênio em fibrina, a trombina ativa fator XIII (FXIIIa) que de forma covalente faz pontes entre fibrinas tornando a coágulo ainda mais estável ${ }^{1,10}$.

$\mathrm{O}$ rFVIIa age ativando diretamente o FX resultando em formação independente de trombina no local (burst de trombina) e contribuindo dessa forma para formação de coágulo estável de fibrina sem a ação dos FVIII e FIX $^{1,4,9,10,11,19}$. O rFVIIa atua ligando-se ao fator tecidual no local da injúria vascular formando um complexo que estimula a formação de trombina (burst de trombina ) pela ativação direta de FX e FIX na presença de plaqueta ativada ${ }^{1,5,6,10,17}$. Um mecanismo alternativo descrito pelos autores é a ativação de FX mediada pelo rFVIIa e subsequente formação de trombina, independente de fator tecidual, na presença de quantidade suficiente de fosfolípede na superfície das plaquetas ativadas ${ }^{1,6,10,17}$. Ambos autores observaram ainda que o burst de trombina contribui para aumento da adesividade e agregação plaquetários, o que pode explicar a ação da medicação em situações de trombocitopenia ${ }^{1}$. Dessa forma, a ação farmacológica 
do rFVIIa induz formação de trombina e contribui para estabilidade da rede de fibrina no local da lesão vascular, resistente à fibrinólise precoce, além de contribuir para ativação plaquetária9,12,13.

$\mathrm{O}$ uso de fator recombinante VII ativado (rFVIIa) na prática médica foi iniciado na década de 80 como agente pró-hemostático ${ }^{2,9}$. A US Food and Drugs Administration (FDA) licenciou o uso do rFVIIa em 25 de março de 1999 para profilaxia e tratamento de episódios de sangramento em pacientes com antecedente de hemofilia A ou B e inibidores de fator VIII e IX, mesmo após uso de fator VIII e IX (USA) (I, $, 3,4,6,7,9,12,17,19$. Tem sido usado com sucesso em pacientes com hemofilia adquirida (anticorpos antifator VIII ou IX), deficiência de fator VII e trombastenia de Glanzmann refratários à reposição plaquetária (União Européia) $)^{1,2,4,9,12,19}$. A dose de rFVIIa nestas situações, varia de acordo com a indicação: $90 \mathrm{mcg} / \mathrm{kg}$ de peso corpóreo a cada duas a três horas para pacientes com hemofilia A e B com inibidores, hemofilia adquirida e trombastenia de Glanzmann; 15 a $30 \mathrm{mcg} / \mathrm{kg}$ de peso corpóreo a cada quatro a seis horas para pacientes com deficiência congênita de fator VII ${ }^{5}$. No mesmo ano, após reportar caso de um soldado israelita com sangramento maciço e de difícil controle no qual foi utilizado rFVIIa com sucesso, seu uso foi ampliado para outras situações clínicas ${ }^{6,17}$. Dessa forma, começaram a ser publicados diversos trabalhos mostrando a eficácia do rFVIIa como agente pró-hemostático em paciente com outras coagulopatias ou pacientes previamente hígidos com história de sangramento agudo de grande monta, principalmente envolvendo trauma, cirurgia cardíaca e hepática, na maioria das vezes com resultado positivo ${ }^{1,4,9,12,17}$.

Trauma é a principal causa de mortalidade no mundo, correspondendo a cinco milhões de casos por ano e representando grande ônus à sociedade por comprometer principalmente indivíduos de faixas etárias economicamente ativas $^{8,14,16,19}$. Sangramento incontrolado continua sendo o principal desafio no atendimento a estes pacientes, correspondendo a $40 \%$ das causas de óbito relacionadas ao trauma, estando associado à coagulopatia difusa em 25 $36 \%$ dos casos de vítimas de trauma, sendo risco independente para mortalidade ${ }^{8,14,16,19}$. O manejo do sangramento nas primeiras horas após o trauma é a principal forma de prevenir o óbito ${ }^{8,16}$. Nos últimos anos foram alcançados grandes avanços no atendimento ao paciente politraumatizado; porém, apesar da combinação de cirurgia, fixação de esterno, damage control e embolização poder controlar sangramento com grande eficácia, alguns pacientes que apresentam sangramento incontrolado desenvolvem coagulopatia necessitando de tratamento específico ${ }^{16,19}$. Reposição de hemocomponentes nem sempre é suficiente para essas situações e o tratamento da coagulopatia secundária ao trauma pode requerer outras medidas ${ }^{16,19}$. Além disso, é bem conhecida a relação entre transfusão maciça de hemocomponentes e aumento da mortalidade (transmissão de patógenos, reação transfusional e lesão pulmonar associada à hemotransfusão) ${ }^{6}$. A coagulopatia pode ser agravada quando associada à hipotermia e acidose, também denominado "tríade letal"16. Após o trauma, se o paciente apresenta ISS (injury severity score) maior que 25 , acidose $(\mathrm{pH}<7,1)$, hipotermia (temperatura $\left.<34^{\circ} \mathrm{C}\right) \mathrm{e}$ pressão sistólica $<70 \mathrm{mmHg}$ há risco crescente de desenvolver coagulopatia ${ }^{16,19}$. A hipotemia torna-se importante à medida que diminui função enzimática, reduz formação de trombina, reduz formação dos plugs plaquetários e de coágulos de fibrina, pois aumenta a degradação do coágulo ${ }^{14}$. Outros fatores contribuem para que ocorra coagulopatia como: perda de sangue, consumo de plaquetas, diluição de plaquetas e fatores de coagulação, aumento da fibrinólise, alteração da função de plaquetas e fatores de coagulação, hipocalcemia, uso de colóides (em acima de 10 unidades de concentrado de hemácias é comum que ocorra coagulopatia e trombocitopenia $)^{6,16}$. O uso de hemocomponentes (concentrado de hemácias e plaquetas, plasma, fibrinogênio, crioprecipitado) é bem estabelecido no atendimento a pacientes com grandes sangramentos, porém não existem guidelines que definam a melhor forma de utilizá-los ${ }^{16}$. Quando o paciente apresenta evidência de sangramento microvascular, hematócrito entre 21 e $24 \%$, tempo de protrombina (TP) ou tempo de tromboplastina parcial ativada (TTPa) maior que 1,5 vezes o normal, trombocitopenia com plaquetopenia menor que 50x109/L, ou concentração de fibrinogênio menor que $1 \mathrm{~g} / \mathrm{L}$, deve ser considerada a hipótese de coagulopatia ${ }^{16,18}$. Esses valores podem representar gatilhos para terapia com hemocomponentes, porém nem sempre a infusão desses restaura a coagulação adequada do paciente ${ }^{16}$. Neste momento a terapia adjuvante com rFVIIa deve ser considerada ${ }^{8,16}$. Além disso, em pacientes com história de traumatismo crânio-encefálico o uso de rFVIIa torna-se importante para controle rápido do sangramento visto que é necessário manter pressão arterial adequada para garantir a perfusão cerebral, ou seja, hipotensão arterial permissiva nestes casos está contra-indicado ${ }^{8}$.

Apesar do trauma ser causa comum de sangramento, pacientes podem sangrar por inúmeras razões, como, por exemplo, doenças envolvendo o fígado ${ }^{18}$. Por ser ele o principal local de síntese e metabolização dos fatores de coagulação dependentes de vitamina K (II, VII, IX, X), síntese de fatores anticoagulantes e componentes envolvidos com o sistema fibrinolítico, como cirrose hepática, podem cursar com alteração na coagulação ${ }^{10,18}$. Pacientes com doença hepática avançada cursam com tempo de protrombina e tempo de tromboplastina parcial ativada alargados, além de plaquetopenia ${ }^{18}$. Apesar da rara ocorrência de sangramento espontâneo, certamente apresentam risco aumentado para ocorrência de sangramento de difícil controle durante qualquer episódio de sangramento ou após procedimento invasivo $^{10,18}$. O manejo do sangramento nestes casos não obteve mudanças importantes nas últimas décadas e se baseia na infusão de plasma fresco congelado (PFC) e concentrado de plaquetas, enquanto infusão parenteral de vitamina $\mathrm{K}$ tem algum benefício apenas em pacientes com colestase prolongada ${ }^{18}$. A coagulopatia nos pacientes com cirrose ocorre por deficiência dos fatores dependentes de vitamina $\mathrm{K}$, sendo o FVII o fator com menor meia-vida, tendo seus níveis como indicativo de prognóstico ${ }^{10}$. O 
uso de rFVIIa tem sido sugerido como opção terapêutica promissora para esses pacientes ${ }^{10,18}$.

Outra causa comum de sangramento no ambiente hospitalar são as operações cardíacas nas quais o uso de bypass cardiopulmonar (up-regulation da expressão de fator tecidual), hemodiluição, disfunção e consumo plaquetários, consumo de fatores de coagulação, hipotermia severa, ativação da cascata de coagulação e fibrinólise, podem agravar o quadro ${ }^{7}$. Nestes casos, sangramento excessivo nas primeiras 24 horas após o procedimento ocorre em $5-7 \%$ dos casos, e o local de sangramento é detectado em menos da metade das ocorrências 7 . Assim como em cirurgia cardíaca, procedimentos envolvendo a área de ginecologia e obstetrícia podem cursar com sangramentos de difícil controle, tendo como causa um grupo heterogêneo de fatores ${ }^{1}$. Nos casos de hemorragia pós-parto (HPP) as causas são: anormalidades placentárias, desordens na coagulação, laceração e trauma, atonia uterina e retenção de conteúdo uterino, cujos fatores de risco englobam anormalidade na posição placentária, gestação avançada, idade maior de 35 anos, obesidade, história de hemorragia pós-parto prévia, pré-eclâmpsia, anemia entre 24 e 29 semanas de gestação antes do parto, desordens na coagulação, instrumentação vaginal durante o parto e trabalho de parto prolongado ${ }^{1}$. Nestes casos o tratamento ainda consiste em grande desafio e muitas vezes torna-se necessário a realização de procedimentos invasivos como histerectomia ou ligadura cirúrgica das artérias ilíacas bilateralmente ${ }^{1}$. A ocorrência de hemofilia A adquirida no pós-parto, ou seja, aparecimento de inibidores de FVIII, também aparece como desafio $^{1}$. A paciente cursa com sangramento excessivo inexplicado ou grandes hematomas em múltiplos sítios associado à alterações laboratoriais: aumento isolado de TTPa associado a TP normal e evidência formal de anticorpos contra VIII, sem que haja antecedente pessoal ou familiar de sangramento ${ }^{1}$. O tratamento convencional para estes casos visa erradicar os anticorpos anti-FVIII com uso de terapia imunossupressora; porém aparecem como opção terapêutica agentes que aumentam os níveis séricos de FVIII, como desmopressina e concentrado de FVIII, e agentes de bypass como concentrado de complexo de protrombina ativada e rFVIIa ${ }^{1}$. Apesar de comumente cursarem com resolução espontânea após aproximadamente 30 meses, nestas situações há altas taxas de morbi-mortalidade ${ }^{1}$. Nos últimos anos houve importante aumento no uso de rFVIIa para essas situações, quando não há resposta a tratamentos convencionais ${ }^{1}$.

O recente aumento do uso de rFVIIa em situações ainda não aprovadas levou ao crescente questionamento sobre eficácia e segurança da medicação em tais situações. Os efeitos colaterais mais observados relacionam-se a eventos tromboembólicos como em pacientes com coagulação intravascular disseminada (CIVD), na qual há expressão sistêmica de fator tecidual, pacientes com placas de aterosclerose, pacientes em sepse e pacientes em uso de drogas antifibrinolíticas ${ }^{6,7,10}$. Postula-se ainda que o rFVIIa contribua para falência de múltiplos órgãos ao modular a resposta inflamatória em pacientes com síndrome da res- posta inflamatória sistêmica (SIRS), que comumente ocorre após cirúrgica cardíaca e uso de bypass cardiopulmonar? Além disso, a dose a ser administrada nestas situações clínicas ainda não está definida ${ }^{7}$. Essas observações têm impulsionado a realização de estudos randomizados, trabalhos clínicos controlados designados a avaliar a eficácia e segurança do rFVIIa em situações clínicas diversas ${ }^{7,10,17}$.

\section{MÉTODO}

Realizou-se pesquisa de artigos nos arquivos eletrônicos PubMed, MedLine e Ovid, usando-se palavras-chave: bleeding, rFVIIa, recombinant factor VIIa, surgery. Os critérios de inclusão utilizados foram: artigos com texto completo disponíveis para leitura; textos publicados em período de 2003 a 2008; artigos com textos completos em inglês ou português. Foram excluídos do estudo artigos de relato de caso. Todos os artigos incluídos foram avaliados e considerados relevantes se incluíssem dados clínicos.

\section{Segurança e eficácia}

O estudo realizado por Goodnough et al. ${ }^{4}$ que avaliou a segurança e eficácia do uso de rFVIIa em situações clínicas ainda não aprovadas, concluiu que são necessários mais estudos para definir a dose e o momento ideal para a administração do rFVIIa; dessa forma o seu uso continua atrelado à decisão pessoal do médico associada ou não à avaliação prévia da equipe de farmacoterapia ou comitê de transfusão do hospital. Em seu estudo o autor avaliou diversas situações, como por exemplo, trauma e oprações complexas nos quais o uso de rFVIIa foi feito na dose de 20 a $120 \mathrm{mcg} / \mathrm{kg}$ apresentando efeito hemostático ideal. Cita o uso pré-cirúrgico de rFVIIa em situações de coagulopatia e neurocirurgia de urgência com resultados positivos normalizando os parâmetros laboratoriais de coagulação após 20 minutos da administração da medicações e sem complicações no peri e pós-operatórios. Ao avaliar o uso de rFVIIa em pacientes submetidos à cirurgia cardíaca, houve benefício tanto na profilaxia quanto no tratamento, com exceção de um trabalho que avaliou o uso profilático da medicação em crianças submetidas à correção de malformação cardíaca congênita, no qual não foi observado benefício. No estudo, uma série avaliada sugere a ineficácia do uso tardio do rFVIIa. Ao avaliar o uso de rFVIIa em pacientes com antecedente de deficiência de FVII; observou excelentes resultados nas séries estudadas e sugere a dose de $20 \mathrm{mcg} / \mathrm{kg}$ para esses pacientes. Da mesma forma, para uso de rFVIIa em doentes que receberam anticoagulante oral evoluindo com prolongamento de INR (international normalized ratio), em todos os casos após uso de 20 a 90 $\mathrm{mcg} / \mathrm{kg}$ de rFVIIa, os resultados foram positivos, sugerindo que o rFVIIa atue adequadamente na reversão da ação de warfarin e outros inibidores de fatores de coagulação dependentes de vitamina $\mathrm{K}$, nos quais a administração de vitamina $\mathrm{K}$ foi insuficiente. Nos estudos envolvendo pacientes com disfunção hepática e os com acidente vascular cerebral hemorrágico, os efeitos do uso de rFVIIa observados foram benéficos. Ao estudar especificamente a 
segurança no uso de rFVIIa, o autor observou que a ocorrência de eventos adversos aparece em menos de um caso a cada 11300 pacientes tratados. Levantamento publicado pela FDA entre 25 de março de 1999 e 31 de dezembro de 2004, avaliando eventos tromboembólicos nos pacientes que receberam rFVIIa em situações clínicas não padrão, indicou um total de 151 eventos tromboembólicos: trombose venosa profunda, acidente vascular cerebral, enfarto agudo do miocárdio, tromboembolismo pulmonar e trombose arterial. Em 38\% dos casos houve uso concomitante de outros agentes hemostáticos e em $72 \%$ de óbito, o rFVIIa foi citado como provável causa. Conclui que são necessários estudos randomizados para avaliar melhor a segurança e eficácia da medicação. Um subsequente trabalho que reportou a análise de segurança do uso de rFVIIa em 13 estudos clínicos envolvendo pacientes com cirrose, trauma e reversão de terapia anticoagulante, o autor não encontrou diferença significativa entre placebo e grupo tratado para a ocorrência de eventos adversos $(P=0,57)$.

Dessa forma, o autor sugere, baseado em seu estudo, que o uso de rFVIIa deve ser feito com cautela para o paciente em estado de hipercoagulabilidade conhecida ou com história de sangramento excessivo com CIVD ou estado de ativação generalizada do sistema hemostático. Com relação à dose da medicação a ser administrada, o autor sugere a de $4,8 \mathrm{mg}$, que corresponde para um adulto com peso entre 50 e $100 \mathrm{~kg}$, a dose de 50 a $100 \mathrm{mcg} / \mathrm{kg}$.

Em meta-análise, Ranucci et al. ${ }^{12}$ avaliou a segurança e eficácia do rFVIIa em pacientes submetidos à grandes procedimentos cirúrgicos e demonstrou diminuição significante no sangramento em pacientes que receberam a medicação. Não houve aumento de eventos tromboembólicos ou mortalidade e nenhum estudo demonstrou diminuição no tempo de internação em UTI ou no tempo total de internação hospitalar. $\mathrm{O}$ autor conclui que o uso profilático de rFVIIa é eficiente em reduzir a taxa de transfusão de concentrado de hemácias alogênico, porém são necessários novos estudos enfocando dose e efeito no uso de rFVIIa.

Johansson6 analisou trabalhos clínicos randomizados e também avaliou eficácia e segurança no uso de rFVIIa para tratamento de hemorragia em situações clínicas não usuais. Analisou situações não cirúrgicas (transplante de células tronco hematopoiéticas, hemorragia intracerebral, dengue hemorrágica, cirrose) e cirúrgicas (prostatectomia, trauma pélvico, transplante hepático, ressecção hepática, cirurgia cardiovascular, correção de cardiopatia congênita, trauma, neurocirurgia, operações em grandes queimados). Nos 17 estudos, apenas quatro tinham resultados positivos quanto à análise da eficácia do rFVIIa e enfatiza que os estudos que mostraram benefício no uso da medicação, foram pequenos e não tiveram seus achados confirmados em grandes estudos randomizados. A diferença na dose e no intervalo entre os trabalhos torna a avaliação da eficácia do rFVIIa difícil, além disso na maioria dos estudos não havia protocolo de transfusão e outros componentes hemostáticos (fibrinogênio, fatores de coagulação e contagem plaquetária) apresentavam valores ideais bastante heterogêneos. De maneira notável, nos estudos que evidenciaram diminuição no sangramento com o uso de rFVIIa, a contagem plaquetária era normal ou o gatilho para transfusão de plaquetas era $75 \times 109 / 1$ ou mais. Em seu estudo, não foi observado aumento de eventos adversos nos paciente tratados com rFVIIa, porém o autor ressalta que não pode ser concluído apenas pelo seu trabalho que não há aumento de eventos tromboembólicos, visto que na maioria dos artigos incluídos, pacientes com antecedentes de fatores de risco para eventos tromboembólicos foram excluídos.

Para Von Heymann et al. ${ }^{5}$, o uso do rFVIIa durante e após procedimento cirúrgico envolvendo região abdominal é seguro e eficaz. Porém, caso não haja registro de coagulopatia prévia, para o autor não há indicação do uso profilático da medicação. Contudo, o rFVIIa deve ser usado como terapêutica adicional em situações de sangramento refratário ao tratamento convencional, ressaltando a importância de evitar o uso tardio da medicação e corrigir desordens que podem comprometer a eficácia da medicação como acidose metabólica (manter $\mathrm{pH}>7,2$ ), hipofibrinogenemia (manter $>100 \mathrm{mg} / \mathrm{dL}$ ), plaquetopenia (manter $>50000 / \mathrm{mcL}$ ) e diminuição do hematócrito (manter $>24 \%$ ). Neste trabalho, a segurança foi clinicamente suficiente, porém observou-se complicações tromboembólicas relacionadas ao uso de rFVIIa associado ao uso de concentrado de complexo de protrombina ativada (aPCCs), ou seja, o aumento da ocorrência de eventos tromboembólicos tornase suspeito devendo ser avaliado o risco-benefício do uso de rFVIIa em pacientes em uso de medicações procoagulantes, mas não caracteriza uma contra-indicação formal.

Em seu artigo de revisão sobre a segurança do uso do rFVIIa em pacientes com coagulopatia secundário na terapia anticoagulante, cirrose e trauma no qual foram avaliados 13 trabalhos clínicos compreendendo 1178 pacientes, Levi et al.10 evidenciou associação significativa entre exposição ao rFVIIA e incidência de eventos trombóticos, embora em muitos estudos haviam pacientes com alto risco de tromboembolismo.

\section{Trauma}

No estudo realizado por Rossaint et al. ${ }^{16}$ foi avaliado o tratamento não cirúrgico para pacientes com quadro agudo de sangramento excessivo, principalmente no trauma. Baseado na ocorrência da tríade letal (coagulopatia, acidose e hipotermia) de maneira precoce no pós-trauma, principalmente relacionado à coagulopatia de consumo, o autor ressalta a necessidade da correção específica da coagulopatia, visto que a infusão de hemocomponentes não restaura parâmetros normais de coagulação. Ao avaliar dois estudos recentes randomizados e controlados envolvendo o uso de rFVIIa nestas situações, o autor observou redução importante na necessidade de transfusão de concentrado de hemácias no grupo que usou a medicação quando comparado ao grupo placebo $(P=0,02)$. Além disso, observou no dia 30 pós-trauma, redução significativa na síndrome de angustia respiratória aguda de $16 \%$ no grupo placebo para $4 \%$ no grupo tratado com rFVIIa $(P=0,03)$. Nos dois estudos não foi observado aumento de eventos adversos, porém o autor ressalta a necessidade de novos trabalhos 
enfocando segurança no uso de rFVIIa, visto que o FDA assinala relação estreita entre o uso de rFVIIa e aumento de eventos tromboembólicos quando o uso da medicação se faz concomitante a outras medicações ou condições clínicas pré-existentes que podem funcionar como fator confundidor.

Rizoli et al. ${ }^{14}$ ao avaliar o uso de rFVIIa em pacientes submetidos a procedimentos cirúrgicos após trauma, cita o primeiro e único trabalho randomizado e controlado com 301 pacientes, 143 com trauma fechado e 134 com ferimento penetrante. Ao analisar todos os pacientes, não foi observada diferença significativa entre os grupos, porém ao excluir pacientes que evoluíram a óbito precocemente, o rFVIIa diminuiu a necessidade de transfusão de concentrado de hemácias nos pacientes com trauma fechado, mas não nos pacientes com ferimento aberto. Apesar das limitações, o autor conclui que esse estudo é a melhor evidência do uso de rFVIIa em trauma, porém ressalta a necessidade de novos estudos para melhor avaliação. $\mathrm{O}$ autor narra que o rFVIIa deve ser usado em pacientes que mantêm sangramento incontrolado após trauma apesar de correção de hipotermia, coagulopatia, trombocitopenia e hemostasia cirúrgica.

Ao analisar dois artigos duplo-cego e randomizados sobre rFVIIa como terapia adjuvante para controle de sangramento em pacientes com trauma severo com coagulopatia, Rizoli et al. ${ }^{13}$ mostra diferença significativa em relação ao grupo controle nos pacientes que sobreviveram mais que 48 horas, em relação ao número de concentrado de hemácias, plasma fresco congelado, plaqueta e transfusão maciça ( mais que 20 concentrados de hemácias). Como os pacientes que receberam rFVIIa foram menos transfundidos em seguimento de 30 dias, na relação ao grupo placebo tiveram significativamente menos falência de múltiplos órgãos e síndrome da angústia respiratória aguda. A limitação deste estudo foi que a definição de coagulopatia (pacientes que receberam oito unidades de concentrado de hemácias) é limitada e imperfeita, pois pacientes com coagulopatia, mas que não foram tratados com hemoderivados, foram excluídos da análise. A despeito dessas limitações o autor conclui que rFVIIa é arma terapêutica valiosa no manuseio do trauma severo, ajudando a deter a hemorragia que ameaça a vida do paciente, reduzindo transfusão de hemoderivados e riscos associados e tendo impacto sobre a morbidade e mortalidade a longo termo associado com sangramento traumático.

Levi et al. ${ }^{9}$ em um estudo placebo controlado em 301 pacientes com trauma fechado e/ou penetrante mostrou redução significativa de necessidade transfusional em pacientes que receberam rFVIIa ( $400 \mathrm{mcg}$ em $4 \mathrm{~h})$ e também tendência de redução na incidência de falência de múltiplos órgãos e síndrome da angústia respiratória aguda.

Kluger et al. ${ }^{8}$ em seu estudo sobre segurança do uso do rFVIIa em pacientes politraumatizados com traumatismo craniano mostrou que não houve diferença significativa em relação à mortalidade, média de dias livres de unidade de terapia intensiva, dias livres do ventilador, eventos tromboembólicos e eventos adversos graves entre os pacientes que receberam $400 \mathrm{mcg}$ (hora 0: 200, hora 1: 100, hora 3: $100)$ de rFVIIa e o grupo placebo no período de 30 dias. No estudo o autor ressalta que pacientes politraumatizados com traumatismo craniano constituem grupo especial, pois frequentemente têm prognóstico pobre, alto risco de desenvolver coagulopatia e eventos tromboembólicos. No seu tratamento é importante manter estabilidade hemodinâmica para que seja mantida perfusão cerebral, controle da coagulopatia, e se possível prevenir expansão da hemorragia cerebral. Por estas razões o rFVIIa é importante arma terapêutica desde que não traga eventos adversos e aumento da mortalidade.

\section{Cirurgia cardíaca}

Para Rizoli et al. ${ }^{13}$ os estudos avaliados em cirurgia cardíaca demonstram resposta positiva após uso de rFVIIa nesta situação.

Romagnoli et al. ${ }^{15}$, estudaram o uso de baixas doses de rFVIIa em cirurgia cardíaca $(1,2 \mathrm{mg}$ - a dose mais baixa usada nos EUA), por ser economicamente atraente e observaram redução significante no sangramento pós-operatório, no tempo de internação em UTI, na necessidade de infusão de concentrado de hemácias, plaquetas, PFC, redução na necessidade de re-abordagem cirúrgica e na taxa de mortalidade dos pacientes tratados. Houve aumento na taxa de eventos adversos nos pacientes que receberam rFVIIa $(P=0,006)$, porém foram identificados fatores de risco prévios ao uso do rFVIIa.

Karkouti et al. ${ }^{7}$, ao avaliarem especificamente os efeitos adversos do uso terapêutico de rFVIIa em pacientes submetidos à operação cardíaca, observou diferenças entre o uso precoce ou tardio da medicação. Inicialmente houve aumento dos eventos adversos no grupo que recebeu rFVIIa tardiamente, ou seja, pacientes em que a primeira dose de rFVIIa foi administrada após uso de 8 ou mais unidades de concentrado de hemácias, o que não foi observado no grupo que recebeu a medicação precocemente e no grupo controle. Porém, ao considerar fatores associados ao uso de rFVIIa como idade, peso, sexo, duração e desmame de bypass cardiopulmonar, doença cerebrovascular e total de unidades de concentrado de hemácias utilizados durante internação, não foi observado aumento na ocorrência de eventos adversos, mesmo no grupo que recebeu o rFVIIa tardiamente. Vale ressaltar, que apesar deste achado, o autor sugere que, para pacientes com sangramento refratário, o uso precoce de rFVIIa é benéfico quando comparado ao uso tardio. Para tal afirmação o autor se baseia no risco de pacientes com sangramento refratário evoluírem com instabilidade hemodinâmica e CIVD, aumentando os riscos de eventos adversos com o uso de rFVIIa. Não foi observada diferença estatisticamente significante na taxa de mortalidade entre os grupos.

Meta-análise de Ranucci et al. ${ }^{12}$ avaliou o uso de rFVIIa em pacientes submetidos a grandes procedimentos cirúrgicos, e o cenário mais verificado foi o das cirurgias cardíacas onde observou-se benefício no uso da medicação. 


\section{Doença hepática prévia ou cirurgia hepática}

Tsochatzis et al. ${ }^{18}$ avaliou o uso de rFVIIa em cinco pacientes com antecedentes de cirrose hepática e coagulopatia severa. O uso profilático foi realizado em três pacientes com sucesso; a dose variou de 34 a $48 \mathrm{mcg} / \mathrm{kg}$ e não foram observados eventos adversos. Em dois pacientes o uso terapêutico do rFVIIa em dose baixa $(45-60 \mathrm{mcg} / \mathrm{kg}$ ) também mostrou-se eficiente no controle de sangramento agudo. Reforça-se assim que a dose do rFVIIa para tratamento e profilaxia de sangramento em pacientes com cirrose ainda não é estabelecida pela literatura e nem mesmo sabe-se a farmacocinética da infusão do rFVIIa em pacientes com sangramento. Dessa forma, conclui que são necessários novos estudos para determinar a eficácia e custo-benefício do uso de rFVIIa em pacientes com cirrose, devendo ser usado como adjuvante em estado de hipocoagulabilidade associado a sangramento de difícil controle.

Rizoli et al. ${ }^{14}$ concluiu que apesar dos primeiros estudos demonstrarem sucesso no uso de rFVIIa para pacientes submetidos à operações hepáticas, qautro trabalhos randomizados e controlados recentes falharam em mostrar benefício no uso da medicação. Porém, o autor reforça a necessidade de novos estudos, visto que há mecanismo biológico sólido que justifica a expectativa do rFVIIa ser clinicamente relevante nestas situações.

Gala et al. ${ }^{3}$ estudou os benefícios do uso do rFVIIa em sete pacientes submetidos à transplante hepático complicado e observou resultado positivo em todos os casos, ou seja, foi alcançado melhora significante dos parâmetros laboratoriais de coagulação após 30 minutos da administração da medicação (50-120 mcg/kg, em bolus), associado à melhora clínica. $\mathrm{O}$ autor sugere também que a administração precoce da medicação cursa com melhores resultados. Dessa forma, a melhora observada possibilitou que o tratamento definitivo fosse empregado com segurança, sem que houvesse eventos adversos ou óbito neste estudo. Todavia, ressalta a importância de novos estudos para avaliar os benefícios do tratamento, o momento ideal de administrá-lo, os riscos potenciais e o custo-benefício de seu uso.

Levi et al. ${ }^{9}$ em revisão sistemática da literatura avaliou doença hepática e transplante hepático. Na doença hepática, estudo placebo-controlado em 245 pacientes com cirrose hepática e hipertensão portal apresentando hemorragia digestiva alta mostrou que nos pacientes que usaram rFVIIA (100 mcg $/ \mathrm{kg}$ ) houve redução (7,9\% vs $15,4 \%)$ dos pacientes que não conseguiram controlar o sangramento. Esta redução só foi vista em pacientes Child B e C. No transplante de fígado, estudo placebo controlado não mostrou redução nas necessidades transfusionais em pacientes que receberam rFVIIA na dose $80 \mathrm{mcg} / \mathrm{Kg}$. Em outro estudo placebo controlado em pacientes que receberam rFVIIA na dose de $80 \mathrm{mcg} / \mathrm{Kg}$ submetidos à hepatectomia parcial mostrou tendência à menor perda sanguínea e menor proporção de pacientes que receberam transfusão $(P=0,05)$

Ranucci et al. ${ }^{12}$ ao avaliar o uso do rFVIIa em pacientes submetidos a grandes procedimentos cirúrgicos, observou que nas operações hepáticas os dados são variados. $\mathrm{Na}$ maioria dos trabalhos avaliados, houve diminuição sig- nificante no sangramento em pacientes que receberam a medicação, porém em dois grandes estudos controlados e randomizados não foi observado impacto na perda total de sangue ou no uso de hemocomponentes.

\section{Outros procedimentos cirúrgicos}

Rizoli et al. ${ }^{14}$ estudou especificamente o uso de rFVIIa em pacientes submetidos a procedimentos cirúrgicos. O autor avaliou separadamente diferentes situações em neurocirugia, cirurgia ortopédica e cirurgia obstétrica/ginecológica. Na neurocirurgia, os estudos recentes mostraram relevância no uso de rFVIIa tanto para acidente vascular encefálico hemorrágico quando para outros tipos de procedimentos. Na cirurgia ortopédica e também urológica e vascular, o autor refere que, apesar da pouca experiência na literatura, os trabalhos mostram resultados positivos no uso de rFVIIa em cirurgia urológica e vascular e resultados divergentes na ortopédica. Na cirurgia obstétrica e ginecológica, apesar do autor ter avaliado apenas relatos de caso, séries de caso e resumos apresentados em anais, os resultados observados foram positivos.

Dessa forma, o autor conclui que os recentes resultados positivos descritos na literatura encorajam novos estudos mais rigorosos para definir a real função do rFVIIa em cirurgia. Porém, reforça que alguns princípios já estão estabelecidos, como o fato do rFVIIa ser medicação adicional e não substituta das medicações em uso atualmente. Além disso, sabe-se que o rFVIIa deve ser usado em pacientes que mantêm sangramento incontrolado após trauma apesar de correção de hipotermia, coagulopatia, trombocitopenia e hemostasia cirúrgica. Finaliza concluindo que novas pesquisas são necessárias para definir benefício, segurança, dose, custo benefício e outros aspectos relacionados à infusão de rFVIIa.

A revisão de literatura realizada por Franchini et al. ${ }^{1}$ visou avaliar o uso de rFVIIa em hemorragia relacionada a procedimentos de ginecologia e obstetrícia. Nos casos de hemorragia pós-parto, que englobam um grupo heterogêneo de causas de sangramento, o autor encontrou 65 mulheres que foram tratadas com rFVIIa observando resultado positivo com o uso da medicação. Porém, ressalta a importância ao interpretar tal resultado, pois os trabalhos avaliados não foram controlados. Avalia ainda o uso de rFVIIa em pacientes com hemofilia A adquirida no pósparto, apesar da pequena casuística, em todos os casos a medicação foi utilizada com sucesso. Nos casos de cirurgia ginecológica associadas a sangramento de difícil controle, $o$ autor descreve um total de 14 pacientes incluídas em quatro diferentes trabalhos e observou benefício no uso de rFVIIa em todos. Conclui que os dados na literatura sobre o uso de rFVIIa em ginecologia e obstetrícia são limitados, porém afirma que seu trabalho aponta o rFVIIa como alternativa terapêutica nesses pacientes ou sangramento severo após procedimento ginecológico que não responde às medidas convencionais. Ressalta a importância de estudos controlados para determinar dose e custo-benefício de seu uso, além de riscos potenciais. Não foram observados eventos adversos, porém recomenda-se no trabalho cuidado no 
uso de rFVIIa para estas situações pelo alto risco da ocorrência de eventos tromboembólicos, ativação sistêmica de coagulação e cânceres ginecológicos.

Para Friederich et al. ${ }^{2}$, o uso de rFVIIa em pacientes submetidos a prostatectomia retropúbica mostrou-se seguro e eficaz com relação à diminuição de perda sanguínea e necessidade de hemotransfusão. Observou-se neste estudo redução dose-dependente na taxa de sangramento no período peri-operatório. Além disso, o tratamento com dose de $40 \mathrm{mcg} / \mathrm{kg}$ de rFVIIa (maior dose avaliada no estudo), eliminou a necessidade de transfusão de concentrado de hemácias em todos os pacientes, enquanto mais da metade dos do grupo placebo necessitou do hemocomponente. Observou-se ainda neste estudo que a administração de rFVIIa para pacientes previamente hígidos resulta na formação de trombina que pode ser bloqueada pelos inibidores do complexo fator tecidual/FVIIa. Não foram observados efeitos adversos neste estudo.

Meta-análise de Ranucci et al. ${ }^{12}$ avaliou o uso do rFVIIa em pacientes submetidos a grandes procedimentos cirúrgicos. Em ginecologia e obstetrícia os resultados são favoráveis, assim como em transplante renal, cirurgia pediátrica, cirurgia geral e neurocirurgia. Falhas são descritas no uso de rFVIIa em cirurgia ortopédica. O autor conclui que o uso profilático de rFVIIa é eficiente em reduzir a taxa de transfusão de concentrado de hemácias alogênico, porém são necessários novos estudos enfocando dose e efeito no uso de rFVIIa.

\section{CONCLUSÃo}

O uso de rFVIIa em pacientes com sangramento maciço agudo é medida de salvamento, ou seja, não deve ser utilizado como de escolha. Em muitos estudos analisados, observou-se redução no uso de transfusão de concentrado de hemácias nos pacientes que receberam rFVIIa comparados aos grupos controle que não receberam rFVIIa sem que houvesse aumento nas taxas de mortalidade e eventos tromboembólicos. Porém, outros estudos não mostraram os mesmos achados.

Ainda não há consenso na literatura quanto à dose ideal a ser utilizada nestes pacientes, nem mesmo quanto à forma de administração (bolus dose única $\mathrm{x}$ bolus em doses seriadas) e o momento ideal de administração.

Portanto, ainda se faz necessária a realização de novos estudos a fim de determinar o intervalo de dose ideal para os pacientes com grandes sangramentos secundários a eventos agudos e protocolos para determinar modo de infusão e momento ideal da infusão da medicação para se alcançar o objetivo de seu uso com segurança e eficácia para todos os pacientes.

Vince FAH, Brandão MJN. Use of recombinant activated factor VII for treatment and prophylaxis of major bleeding. ABCD Arq Bras Cir Dig 2009;22(3):171-8

ABSTRACT - Introduction - Recombinant activated factor VII (rFVIIa) is a protein produced by genetic engineering, the structure is very similar to the structure of intrinsic activated factor VII (FVII). Its action is based on knowledge of the coagulation mechanism in vivo by acting in direct activation of factor X independent resulting in formation of thrombin at the injury site and thereby contributing to the formation of stable fibrin clots without the action of factor VIII and factor IX. Methods - Was conducted extensive review of the literature in order to determine the new findings related to the use of recombinant activated factor VII in patients with severe bleeding. Results - It was found that the use of rFVIIa started in the 80's for prophylaxis and treatment of bleeding in patients with a history of hemophilia A or B with inhibitors to factor VIII and IX, factor VII deficiency and Glanzmann's thrombasthenia refractory to replacement platelet. In 1999 its use was expanded to other clinical situations and thus began to be published several studies showing the efficacy of rFVIIa as a pro-hemostatic agent in patients with bleeding disorders or other previously healthy patients with a history of acute bleeding of major consequence. Trauma is the leading cause of mortality worldwide and uncontrolled bleeding the main challenge in caring for these patients. It is common for the association of trauma with coagulopathy, requiring in some cases specific therapy to treat it. At this point in adjuvant therapy with $\mathrm{rFVII}$ a should be considered. Other common causes of bleeding are the heart, gynecologic/obstetric surgeries and diseases involving the liver. The coagulopathy in these cases is deficiency of factors dependent on vitamin K, and the FVII factor with smaller half life. Conclusion - The use of rFVIIa has been suggested as a promising therapeutic option for these patients. Thus, the recent increase in the use of rFVIIa in situations not approved has led to growing questions about efficacy and safety of this specific medication in such situations.

HEADINGS - Factor VII. Bleeding.

\section{REFERÊNCIAS}

1. Franchini M, Lippi G, Franchi M. The use of recombinant activated factor VII in obstetric and gynecological haemorrhage. BJOG 2007; 114:8-15;

2. Friederich PW, Henny CP, Messelink EJ, Geerdink MG, Keller T, Kurth KH, Buller HR, Levi M. Effect or recombinant activated factor VII on perioperative blood loss in patients undergoing retropubic prostatectomy: a double-blind placebo-controlled randomized trial. Lancet 2003; 361:201-205;

3. Gala B, Quintela J, Aguirrezabalaga J, Fernández C, Fraguela J, Gómez M. Benefits of Recombinant Activated factor VII in Complicated Liver Transplantation. Trans Proceed 2005; 37:3919-3920;
4. Goodnought LT, Shander AS. Recombinant Factor VII: safety and efficacy. Curr Opin Hematol 2007; 14:504-509;

5. Heymann CV, Jonas S, Spies C, Wernecke KD, Ziemer S, Janssen D, Koscielny J. Recombinant activated factor VII for the treatment of bleeding in major abdominal surgery including vascular and urological surgery: a review and meta-analysis of published data. Crit Care 2008; 12:1214;

6. Johansson PI. Off-label use of recombinant factor VIIa for treatment of haemorrhage: results from randomized clinical trials. Vox sanguinis 2008; 95: 1-7; 
7. Karkouti K, Yau TM, Riazi S, Dattilo KM, Wasowicz M, Meineri M, McCluskey SA, Wijeysundera DN, Rensburg AV, Beattie WS. Determinants of complications with recombinant factor VIIa for refractory blood in cardiac surgery. Can J Anesth 2006; 53:802-809;

8. Kluger Y, Riou B, Rossaint R, Rizoli SB, Boffard KD, Choong PIT, Warren B, Tillinger M. Safety of rFVIIa in hemodinamically unstable polytrauma patients with traumatic brain injuri: post hoc analysis of 30 patients from a prospective, randomized, placebo-controlled, double-blind clinical trial. Crit Care 2007; 11:R85;

9. Levi M, Peters M, Buller HR. Efficacy and safety of recombinant factor VIIa for treatment of severe bleeding: A systematic review. Crit Care Med 2005; 33:883-890;

10. Levy J, Fingerhut A, Brott T, Langbakke I, Erhardtsen E, Porte RJ, Recombinant factor VIIa in patient with coagulopathy secondary to anticoagulant therapy, cirrhosis, or severe traumatic injury: review of safety profile. TRANSFUSION 2006; 46:919-933;

11. Protocolo de indicação de rFVIIa em pacientes não hemofílicos; Hemocentro da Faculdade de Ciências Médicas da Campinas - UNICAMP, 2007.

12. Ranucci M, Isgrò G, Soro G, Conti D, De Toffol B. Efficacy and Safety Recombinant Activated Factor VII in Major Surgical Procedures. Arch Surg 2008; 143 (3):296-304;

13. Rizoli SB, Boffard HD, Riou B, Warren B, Ian P, Kluger Y, Rossaint R, Tillinger M, Novoseven Trauma Study Group. Recombinant activated factor VII as an adjunctive therapy for bleeding control in severe trauma patients with coagulopathy: subgroup analysis from two randomized trials. Crit Care 2006; 10:R178;
14. Rizoli SB, Junior BN, Osman F, Netto FS, Kiss A, Callum J, Brenneman FD, remblay L, Tien HC. Recombinant Activated Coagulation Factor VII and Bleeding Trauma Patients. J Trauma 2006; 61:1419-1425;

15. Romagnoli S, Bevilacqua S, Gelsomino S, Pradella S, Ghilli L, Rostagno C, Gensini GF, Sorbara C. Small-Dose Recombinant Activated Factor VII (NovosevenR) in Cardiac Surgery. Anesth Analg 2006; 102:1320-6;

16. Rossaint R, Duranteau J, Stahel PF, Sphn DR. Nonsurgical Treatment of Major Bleeding. J An Clin 2007; 12:35-48;

17. Scarpelini S, Rizoli S. Recombinant factor VIIa and surgical patient. Curr Opin Crit Care 2006; 12:351-356;

18. Tsochatzis E, Papatheodoridis GV, Elefsiniotis I, Thanelas S, theodossiodes G, Moulakakis A, Archimandritis AJ. Prophylactic and therapeutic use of recombinant activated factor VII in patients with cirrhosis and coagulation impairment. YDLD 2006; 851:No of pages 5;

19. Vincent JL, Rossaint R, Riou B, OzierY, Zideman D, Spahn DR. Recommendations on the use of activated factor VII as an adjunctive treatment for massive bleeding - a European perspective. Crit Care 2006; 10:R120.

Fonte de financiamento: não há Conflito de interesse: não há Recebido para publicação: 14/02/2009 Aceito para publicação: 22/04/2009 